\title{
Semiparametric binary model for clustered survival data
}

\begin{abstract}
This paper considers a method to analyze semiparametric binary models for clustered survival data when the responses are correlated. We extend parametric generalized estimating equation (GEE) to semiparametric GEE by introducing smoothing spline into the model. A backfitting algorithm is used in the derivation of the estimating equation for the parametric and nonparametric components of a semiparametric binary covariate model. The properties of the estimates for both are evaluated using simulation studies. We investigated the effects of the strength of cluster correlation and censoring rates on properties of the parameters estimate. The effect of the number of clusters and cluster size are also discussed. Results show that the GEE-SS are consistent and efficient for parametric component and nonparametric component of semiparametric binary covariates.
\end{abstract}

Keyword: Clustered survival data; Generalized estimated equation; Simulation; Smoothing spline 